Möbus C., Eilers, M. \& Garbe, H., Predicting the Focus of Attention and Deficits in Situation Awareness with a Modular Hierarchical Bayesian Driver Model, in: Vincent G. Duffy (Ed.), Digital Human Modeling, HCI 2011, Orlando, FL, USA

\title{
Predicting the Focus of Attention and Deficits in Situation Awareness with a Modular Hierarchical Bayesian Driver Model
}

\author{
Claus Möbus $^{1,2}$, Mark Eilers ${ }^{1,2} \&$ Hilke Garbe \\ Learning and Cognitive Systems / Transportation Systems \\ C.v.O University / OFFIS, Oldenburg, Germany \\ http://www.lks.uni-oldenburg.de/ \\ \{claus.moebus,hilke.garbe\}@uni-oldenburg.de,mark.eilers@offis.de
}

\begin{abstract}
Situation Awareness (SA) is defined as the perception of elements in the environment within a volume of time and space, the comprehension of their meaning, and the projection of their status in the near future [1]. Lacking SA or having inadequate SA has been identified as one of the primary factors in accidents attributed to human error [2]. In this paper we present a probabilistic machine-learning-based approach for the real-time prediction of the focus of attention and deficits of SA using a Bayesian driver model as a driving monitor. This Bayesian driving monitor generates expectations conditional on the actions of the driver which are treated as evidence in the Bayesian driver model.
\end{abstract}

Keywords: Focus of attention, deficits in situation awareness, Bayesian autonomous driver model, Bayesian driving monitor, modular hierarchical Bayesian driver model, learning of action-relevant percepts

\section{Introduction}

Driver's behavior should be regarded as an instance of Cognition in the Wild [3]. Its study is not possible in highly controlled laboratory experiments but only in natural (real or simulated) traffic scenarios. A natural scenario includes all hazards even where they are rare events. Because of their clinical cleanness most simulated traffic scenarios are lacking this kind of naturalness. They are not ecological valid with respect to experiments and cues: the external validity of simulation experiments is doubtful. This is especially important when studying SA. Scenarios without hazards or with a biased set of hazards cause biased theoretical hypotheses and empirical results. In this paper we assume, that all SA-studies which will be planned according to our proposals are ecological valid. Unfortunately, this was not the case for the simulated driving studies discussed here.

${ }^{1}$ project Integrated Modeling for Safe Transportation (IMOST) sponsored by the Government of Lower Saxony, Germany under contracts ZN2245, ZN2253, ZN2366

${ }^{2}$ project ISi-PADAS funded by the European Commission in the 7 th Framework Program, Theme 7 Transport FP7-218552 
Möbus C., Eilers, M. \& Garbe, H., Predicting the Focus of Attention and Deficits in Situation Awareness with a Modular Hierarchical Bayesian Driver Model, in: Vincent G. Duffy (Ed.), Digital Human Modeling, HCI 2011, Orlando, FL, USA

\section{Early Models of Looking and Driving}

There is a wealth of reported eye movement strategies and relevant perceptional cues which are sometimes contradictory or ambiguous [4, ch.7].

When driving on empty straight roads two cues are required for proper lane maintenance: the location relative to the lane edges at some preview distance and the splay angles (Fig. 1) that the lane edges make with the driver's heading [5, 6].

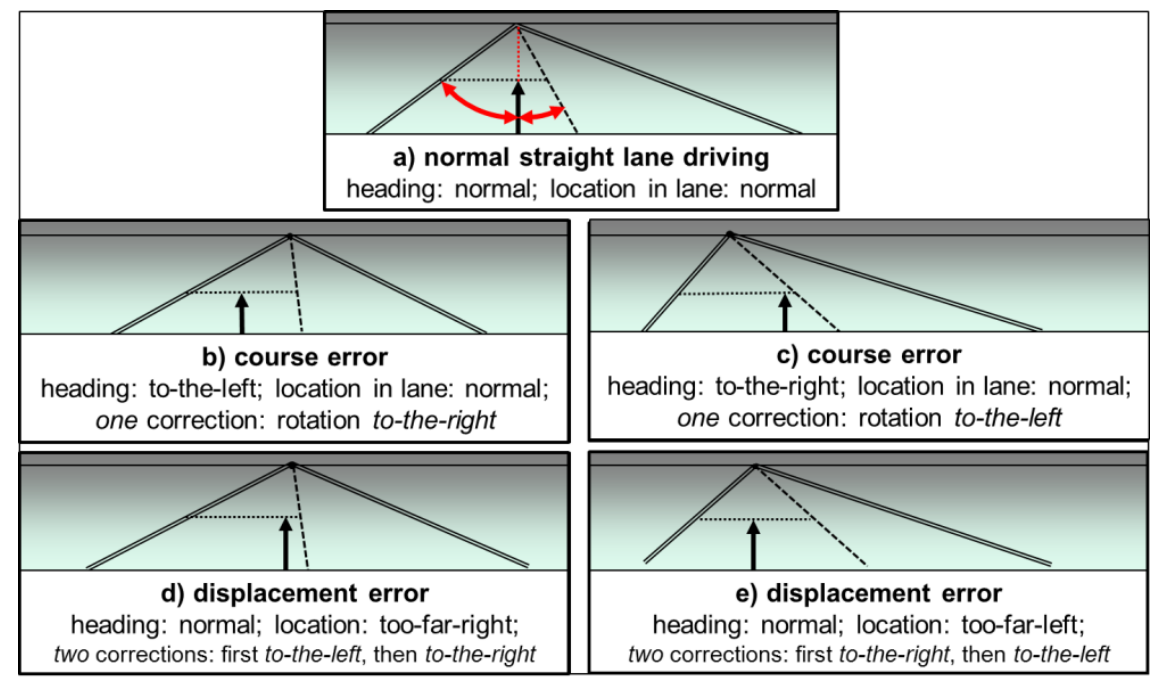

Fig. 1. Steering corrections in the UK (right-hand-drive car in the left lane) as a function of heading (bold arrow), splay angles, and location (displacement) at preview distance (horizontal dotted line; adapted from [4, p.118]). Splay angles in reality differ from those in Fig. 1.

Only one corrective rotation is needed to reduce the risky situation 1.b to the normal position 1.a or from 1.c to 1.a. Two corrections are necessary to reduce $1 . \mathrm{d}$ to 1.b and then to 1.a or to reduce 1.e to 1.c and then to 1.a.

Changing lanes involves a three-stage maneuver: a turn out-of-lane $(1 . a \rightarrow 1 . c)$, a period of oblique travel, and then a counterturn (1.c $\rightarrow 1 . a)$ in the new lane [4]. The quality of the maneuver was reported to improve when a slower vehicle ahead was the reason for the lane change and could be used as an inertial cue for perception [7].

Steering around (gentle) bends is more complicated, because splay angles change with distance and during the drive. The gaze sampling strategy assumes that drivers choose a point on the future path at a constant preview distance and uses the horizontal eccentricity of this point to adjust steering [4, 8, and 9]. The steering wheel angle is a function of the path curvature, which can be estimated by the angle between gaze direction (to a preview point $\mathrm{P}$ at a distance D) and heading. According to flowfield theory the driver samples cues in the form of flow lines from various parts of the view field [4, 8 - 10].

Winding country roads require anticipatory estimates of the future curvature from road features or flow lines. Land \& Lee [11] found that drivers spend much time looking at the tangent point of the upcoming bend. This is the moving point on the 
Möbus C., Eilers, M. \& Garbe, H., Predicting the Focus of Attention and Deficits in Situation Awareness with a Modular Hierarchical Bayesian Driver Model, in: Vincent G. Duffy (Ed.), Digital Human Modeling, HCI 2011, Orlando, FL, USA

inside where the driver's gaze line is tangential to the road edge. It is nearly stationary in the flow field when the curvature is constant. Similar findings in favor of this strategy were reported [12 - 15]. Other studies favor the hypothesis that it is not the tangent point but the future path that is viewed more frequently [16 - 18]. Either way, the steering angle seems to be a function of the gaze angle with a time lag of ca. 0.8 sec [4, Fig. 7.5b]. This seems to be a planned delay to enhance the anticipatory horizon of the driver. When the course is not too demanding there are off-road glances between 0.5 and $1 \mathrm{sec}$ [19] which provide evidence for lateral control with peripheral [20] or ambient [21] vision.

Computational models of steering behavior have been engineered since 1978 [22]. Donges' model uses feedback and feed-forward or anticipatory information from near and distant parts of the road. There is empirical evidence for this particular combination of information [23, 24]. The two-point control model of steering [25] rests on the same combination. The change of the steering wheel angle is a function of the change of the angles between heading and two preview points (near and far point) and of the angle between heading and the near point.

To summarize, there is universal agreement that fluent driving on winding roads requires visual information from both near and distant road regions. The near region involves feedback from the angular position and velocity of the lane boundaries. There is, however, less agreement about which features in the far road region are necessary or sufficient to provide prospective feed-forward information (Mars, 2008) [4, p.129]. During natural driving, the gaze may be intermittently directed to the (tangent point) TP and the road ahead as a way to determine the future path and keep steering smooth at the same time [14].

Other scenarios like braking, overtaking, entering highways, driving in cities, multitasking, etc. provide and require different cues and other more interleaved visual strategies. These are at the moment only rudimentary understood or formalized. So we developed a nearly assumption-free modeling strategy which rests on machinelearning, data mining, stochastic models. One of the few assumptions is that relevant percepts or cues could be learnt on-the-fly by stochastic structure-learning techniques.

\section{Hierarchical Modular Bayesian Autonomous Driver Models}

In our earlier research [26-28] we developed a Bayesian Autonomous Driver (BAD) model based on Dynamic Bayesian Networks proposed in [29] (Fig. 2).

The model assumed that one single model is sufficient to generate actions for longitudinal and lateral control in an arbitrary virtual course. Now, we have a more sophisticated view of human competence. We assume and model skill hierarchies (Fig. 3) of human drivers with a new hierarchical modular probabilistic architecture [30, 31] (Fig. 4). According to our skill hierarchy (Fig. 3) the maneuver Overtaking is partitioned into the three behaviors PassOut, PassCar, and PassIn. If we had reused Land \& Tatler's proposal of partitioning the ChangeLane maneuver we would get a partition of seven behaviors for the Overtaking maneuver. 
Möbus C., Eilers, M. \& Garbe, H., Predicting the Focus of Attention and Deficits in Situation Awareness with a Modular Hierarchical Bayesian Driver Model, in: Vincent G. Duffy (Ed.), Digital Human Modeling, HCI 2011, Orlando, FL, USA

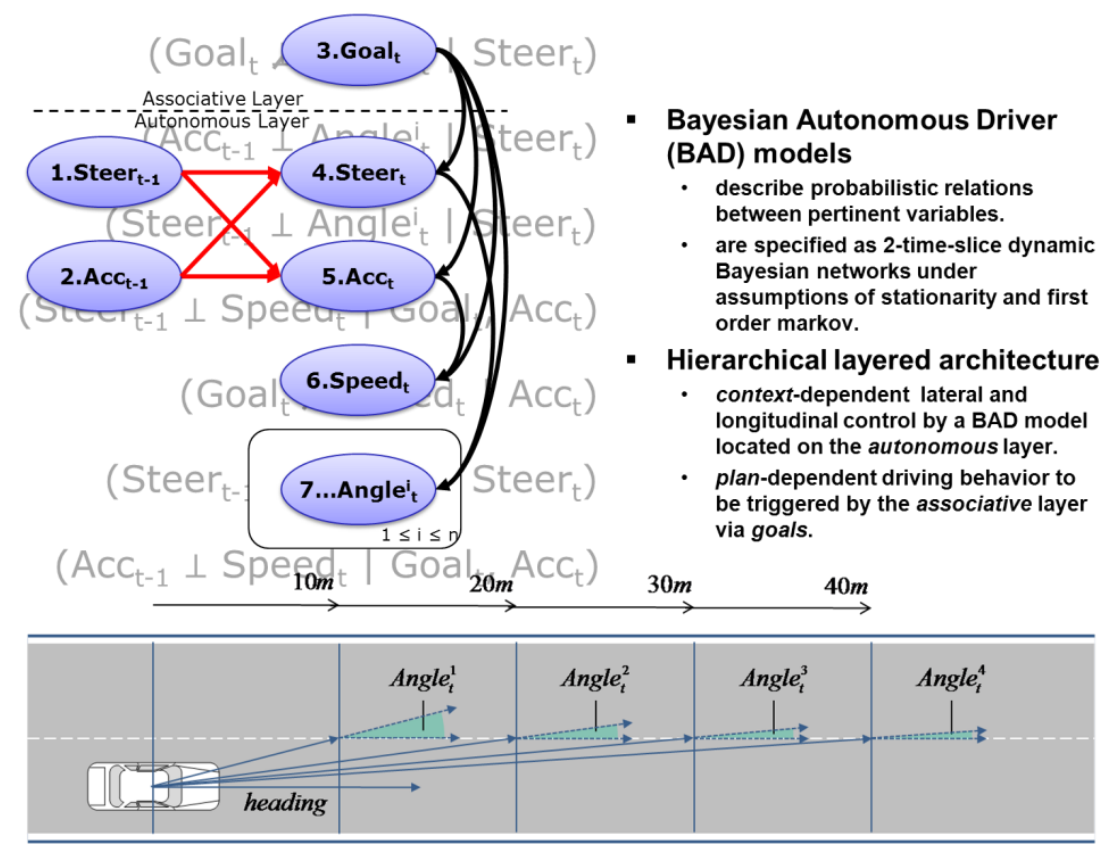

Fig. 2. Reactive Bayesian Autonomous Driver (BAD) model based on a 2-time-sliced dynamic Bayesian network

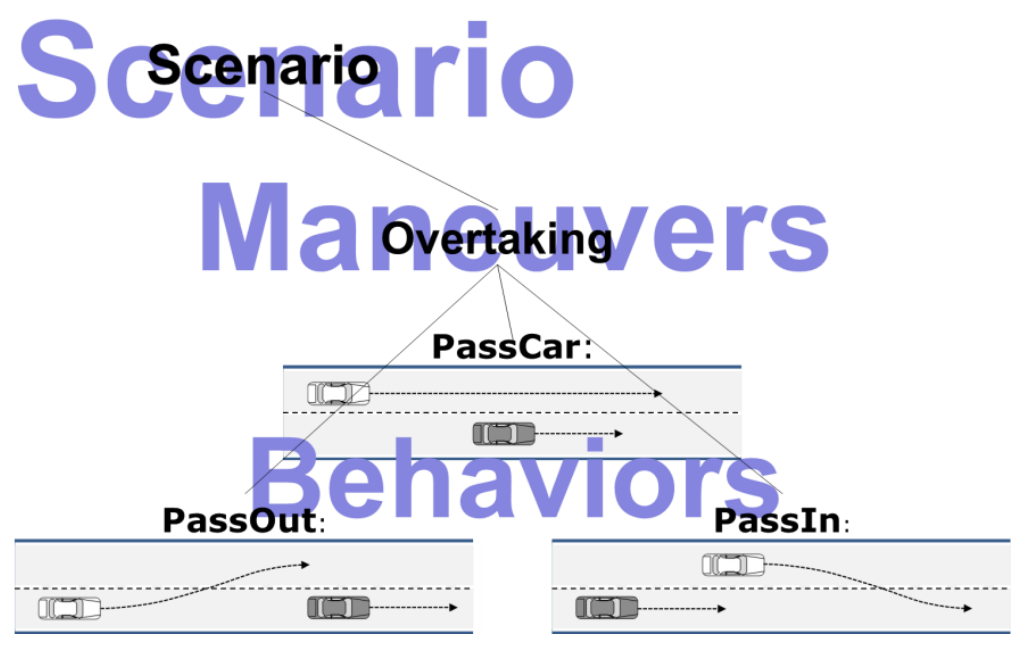

Fig. 3. Skill hierarchy of a driver with partitioning the overtaking maneuver into the three behaviors PassOut, PassCar, and PassIn 
Möbus C., Eilers, M. \& Garbe, H., Predicting the Focus of Attention and Deficits in Situation Awareness with a Modular Hierarchical Bayesian Driver Model, in: Vincent G. Duffy (Ed.), Digital Human Modeling, HCI 2011, Orlando, FL, USA

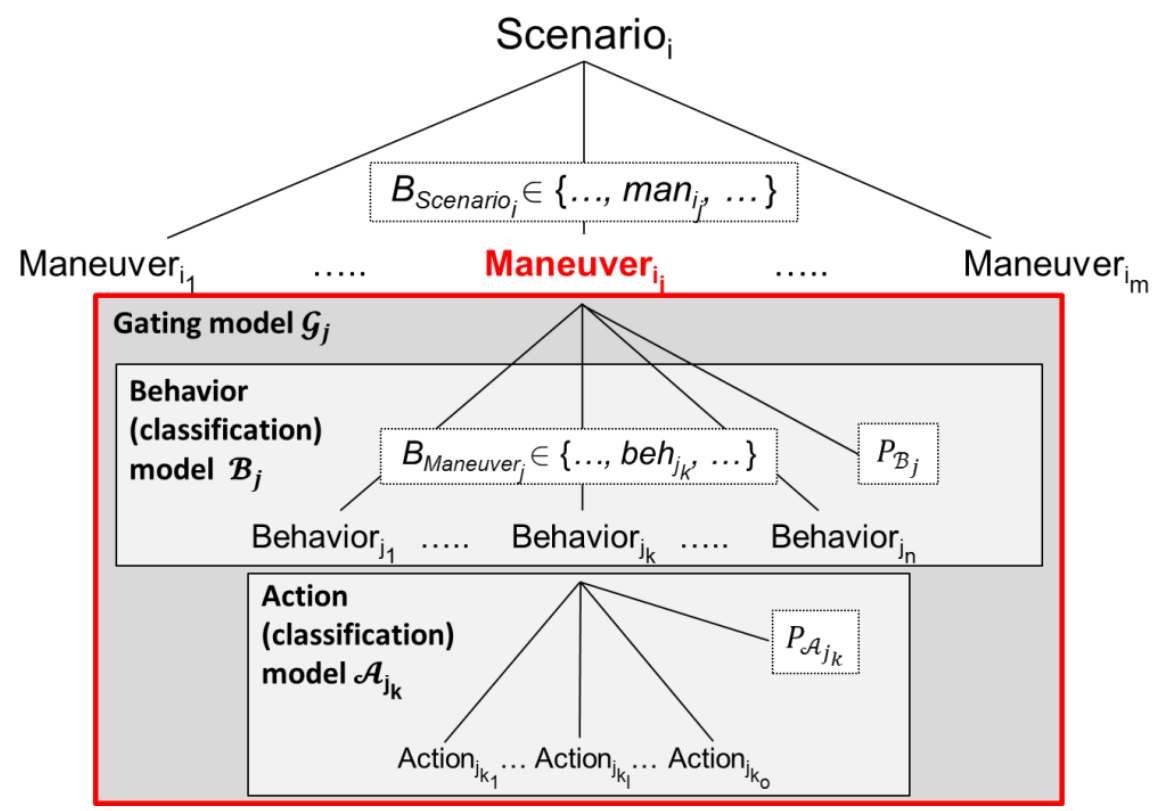

Fig. 4. Implementation of the skill Maneuver ${ }_{j}$ by the assemblage of a Bayesian Gating Model $G_{j}$ and submodels $B_{j}$ and $A_{j k}$ with BIC-relevant peephole percepts $P$

\section{Learning Relevant Percepts in Hierarchical Modular Bayesian Autonomous Driver Models}

Our BAD models are able to drive autonomously in the TORCS simulation environment on a virtual country road - including passing or overtaking maneuvers without opposing traffic (Fig. 5). They rest on the assumption that there is considerable uncertainty about the relevant percepts in natural driving maneuvers. So the relevant percepts have to be identified during the modeling process.

To model the driver's ambient and foveal vision channels [21] we used 205 different time-independent (estimates of speed, distance and angle) and timedependent percepts (TDP). TDPs are percepts similar but not identical to Lee's timeto-X (tau) measures [32-34]. TDP-percepts (e.g. SLA-angles in Fig. 6 and $S C A$-angles in Fig. 7) are defined on points of reference or preview in the scenario. One point of reference is the current position; the points of preview are located in the future course and will be reached by the driver in a certain amount of time depending on the speed of his vehicle. A more detailed description of the variables is given in [31]. This set of variables proved to be informative for lateral and longitudinal control by machinelearning standards.

Single time-dependent angles tended to over-anticipate the upcoming course of the road when approaching sharp curves (e.g. hairpins) with high speed [26]. Fanning out distance sensors from the driver to the street boundaries [28] solved the problem of 
Möbus C., Eilers, M. \& Garbe, H., Predicting the Focus of Attention and Deficits in Situation Awareness with a Modular Hierarchical Bayesian Driver Model, in: Vincent G. Duffy (Ed.), Digital Human Modeling, HCI 2011, Orlando, FL, USA

over-anticipation but were too restrictive and not able to anticipate the course of the road in curves. Now we use sets of time-dependent and-independent angles to encode the vision field of the driver and to avoid the problem of over-anticipating.

In a training phase we selected percepts relevant for the actions steer and delacceleration with a step-wise structure-learning technique using the Bayesian Information Criterion (BIC). We assume - as said before - that the training phase is ecological valid with respect to all hazards. For each skill in the hierarchy the procedure selected a minimal set of relevant ('peephole') percepts $P_{A j k}$ and $P_{B j}$ (Fig. 4, $6,7)$. In total, 41 of the 205 percepts were identified as relevant for Action and Behavior-Classification models [31].

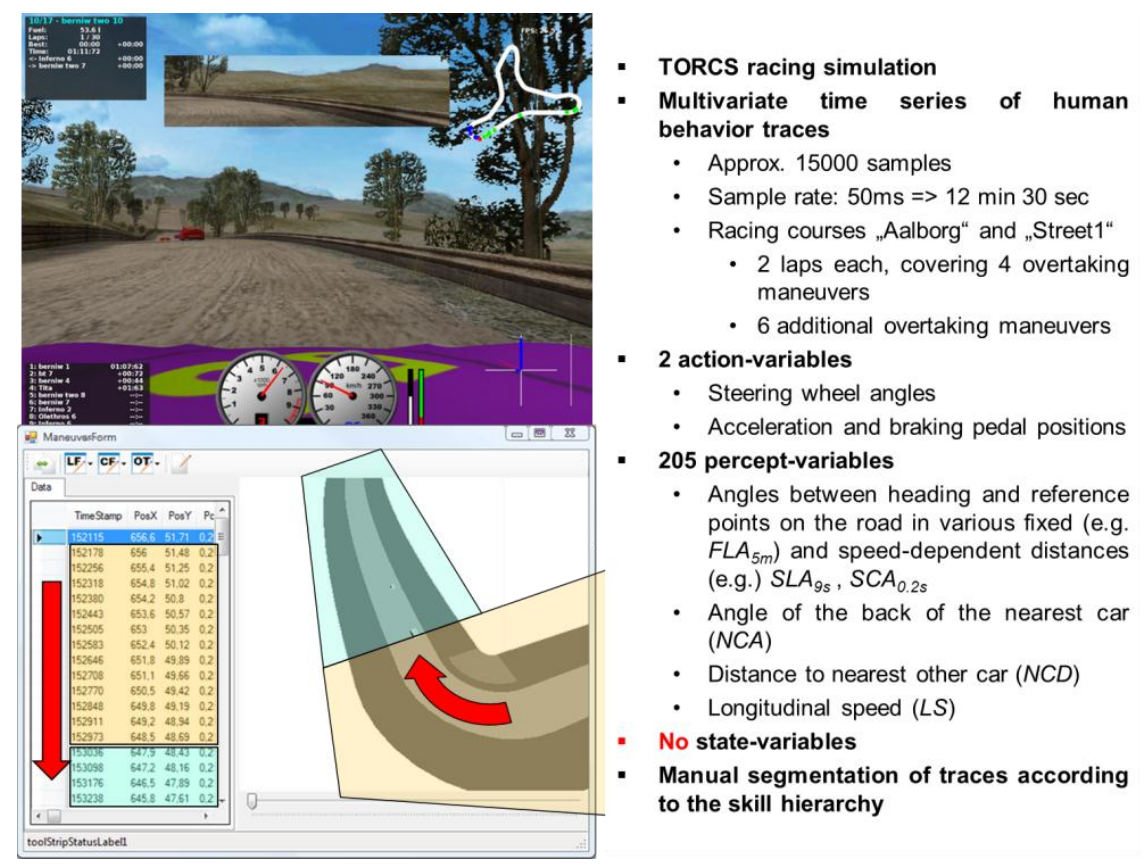

Fig. 5. Experimental setup with TORCS course, variables of interest, and data classification

\section{Predicting the Focus of Attention and Deficits in Situation Awareness with the Bayesian Driver Model as a Driving Monitor}

After the training phase the BAD model could be used as a multi-purpose driving monitor.

First, it could be used to check whether the drive can be considered 'normal' or 'abnormal' according to the standards of a correct selected action model (Fig. 4, 6). This could be checked by computing the likelihood of the drive. The selection of action models is done by the Behavior Classification model (Fig. 4, 7) and its peephole percepts (e.g. $N C A$ and $S L A_{0.2 s}$ in Fig. 7). 
Möbus C., Eilers, M. \& Garbe, H., Predicting the Focus of Attention and Deficits in Situation Awareness with a Modular Hierarchical Bayesian Driver Model, in: Vincent G. Duffy (Ed.), Digital Human Modeling, HCI 2011, Orlando, FL, USA

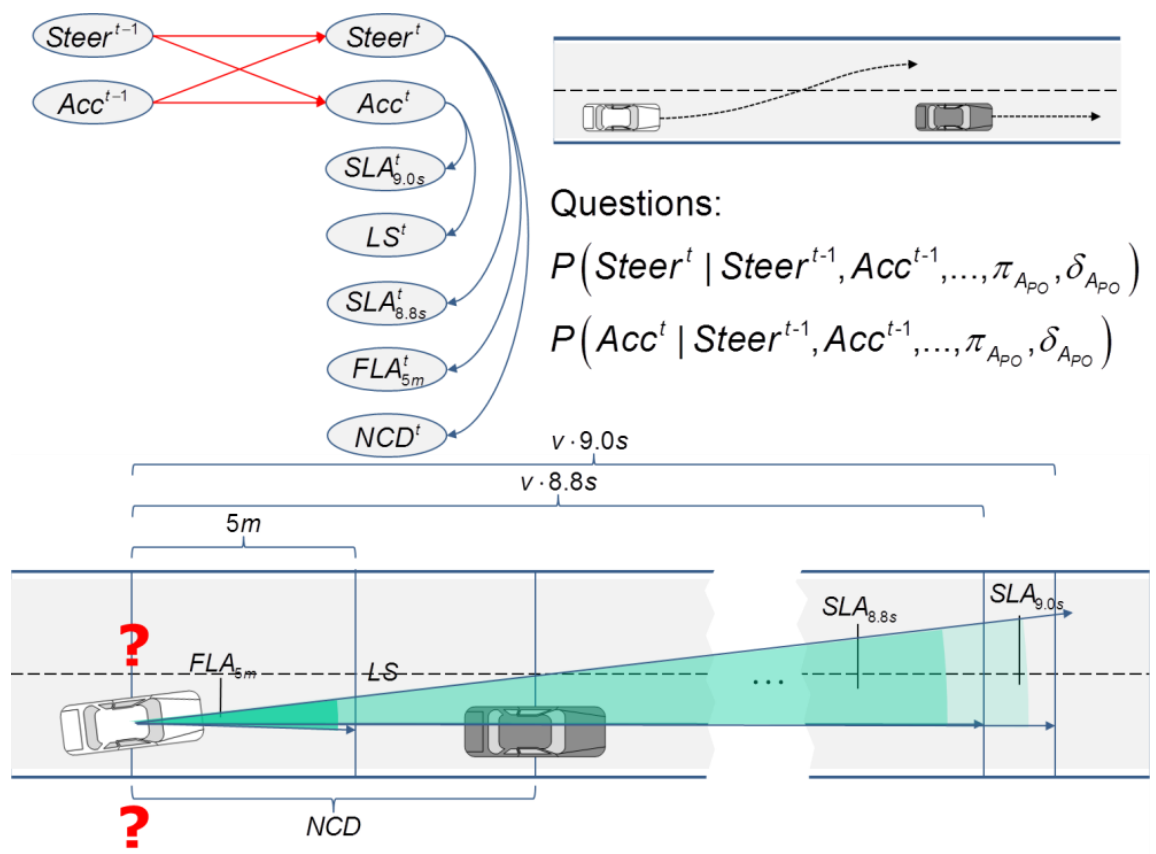

Fig. 6. Action-model PassOut (one speed-, two distance-, and time-based peephole percepts)

- Identification of appropriate mixture of behaviors in a given situation

- Represent behaviors (PassOut, PassCar,

Pass In) by Behavior-variable

- Default structure for efficiency

- 2-time-slice Bayesian networks assuming stationary and first order Markov property

- (Dynamic) naïve Bayesian classifier

- Learning of minimal important (peephole-) percept-variables from human behaviour traces by methods of machine learning

- Heuristic search in the space of all possible combinations of percept-variables

- Score combinations with Bayesian Information Criterion

- Question to identify appropriate behavior:

$$
P\left(B_{O T}^{t} \mid N C A^{t}, S C A_{0.2 s}^{t}, \pi_{B_{O T}}, \delta_{B_{O T}}\right)
$$
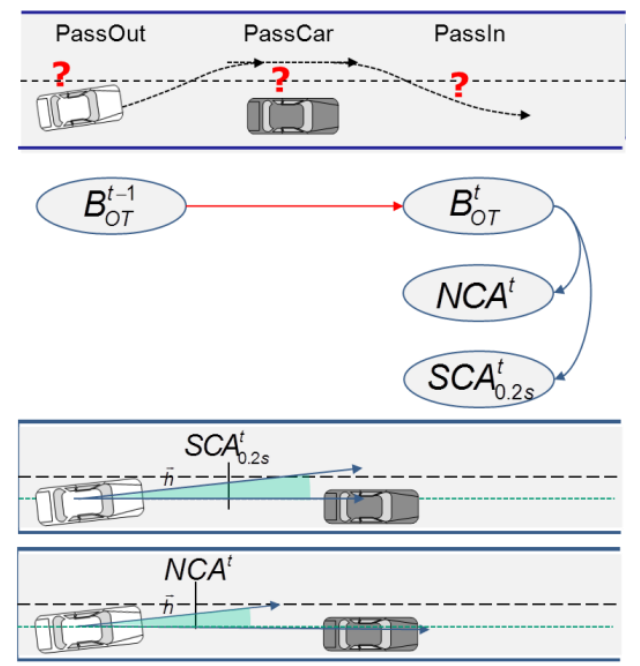

Fig. 7. Behavior-Classification-model Overtaking with one distance-based and one time-based peephole percept 
Möbus C., Eilers, M. \& Garbe, H., Predicting the Focus of Attention and Deficits in Situation Awareness with a Modular Hierarchical Bayesian Driver Model, in: Vincent G. Duffy (Ed.), Digital Human Modeling, HCI 2011, Orlando, FL, USA

Second, predictions of the focus of attention can be obtained. Because the Behavior and the Action-model (Fig. 4) are conceptualized and validated as efficient Bayesian classifier models we are able to predict the relevance or adequacy of percepts on the basis of the driver actions by asking the questions P(Percepts | Actions). In the case of driving the questions are of course reversed in direction to $\mathrm{P}$ (Actions $\mid$ Percepts).

Third, predictions of deficits in situation awareness can be obtained during the same monitoring process. There are two alternatives in SA deficit prediction. In the first case we predict that in certain behaviors or maneuvers some percepts are not relevant in the view of the action model (question marks in Figures 6-9). We expect that we can introduce hazards (e.g. vehicles or pedestrians appearing on the ?-marked sides of the car) which will not be noticed by the driver.

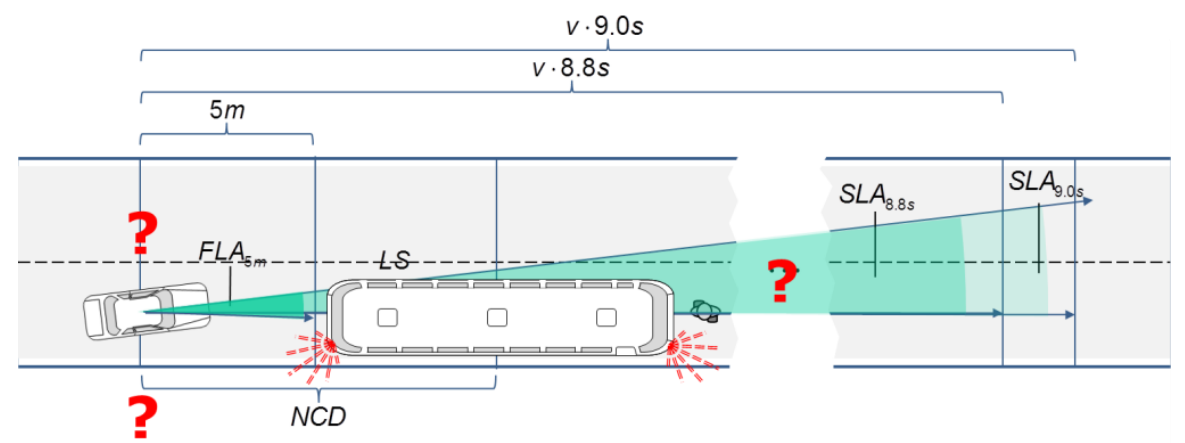

Fig. 8. Inadequate percept-action mapping behavior according PassOut-Action model

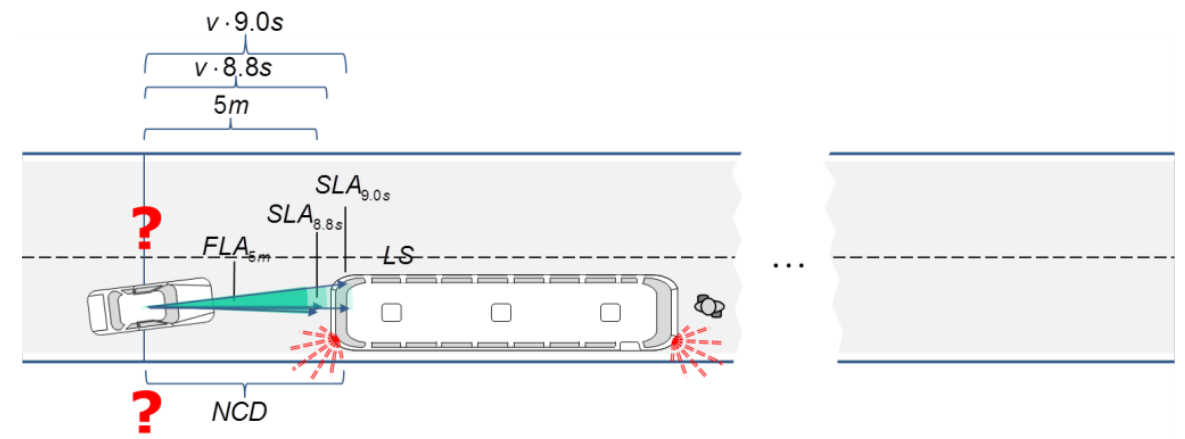

Fig. 9. Adequate percept-action mapping behavior according PassOut-Action model

In the second case the prediction of deficits in SA rests on an abnormality or likelihood check of the driver behavior according to the standards of the selected action model. It is assumed that drivers sometimes choose actions not adequate for the scenario. This could be due to the fact that they substitute real-world percepts by mental assumptions or anticipations. E.g. if we assume that the driver chooses the 
Möbus C., Eilers, M. \& Garbe, H., Predicting the Focus of Attention and Deficits in Situation Awareness with a Modular Hierarchical Bayesian Driver Model, in: Vincent G. Duffy (Ed.), Digital Human Modeling, HCI 2011, Orlando, FL, USA

same speed in Figure 6 and 8 we infer with the help of the driver model, that the relevant percepts are identical.

But in Figure 8 the relevant percepts $S L A$ are inadequate for the chosen behavior because they are shaded by the bus. The driver is driving too fast according to the standards of the PassOut-Action model. An adequate percept-action mapping according to the PassOut-Action model is depicted in Figure 9. A preference for slower driving guarantees that the relevant time-dependent percepts are not "out of sight". Further empirical studies could reveal the necessity of a new PassOutSchoolbus-Action model with a new percept-action mapping (Figure 9).

\section{Summary}

The BAD MoB model could be used as a monitor of the driver's behavior. First, it could be used to compute the likelihood of the actual driving behavior under the assumption of a correct selected action model. Second, it could be used to predict the focus of attention on the basis of driver actions by answering the questions P(Percepts | Actions). Third, it could be used to predict deficits in SA. Behavior and actions which seem to be unlikely in the view of the scenario-relevant valid action model are indicators of reduced SA. At the same time the abducted BIC-relevant percepts should be checked whether they could be really observed in the driving situation. If not then this gives a hint that the driving behavior is inadequate for the situation. Furthermore the abducted nonrelevant percepts give hints where hazards could intrude the local vicinity of the vehicle unnoticed from the situational attention system of the driver.

\section{References}

1. Endsley, M.R.: Toward a theory of situation awareness in dynamic systems. Human Factors 37(1), 32-64 (1995)

2. Horswill, M.S. \& McKenna, F.P.: Driver's Hazard Perception Ability: Situation Awareness on the Road, In: Banbury \& Tremblay (eds), A Cognitive approach to situation awareness: theory and application, Ashgate Publishing, Ltd., p. 155 - 172 (2004)

3. Hutchins, E.: Cognition in the Wild, MIT Press, Cambridge, Mass. (1996)

4. Land, M.F. \& Tatler, B.W.: Looking and Acting: Vision and eye movements in natural behavior, Oxford University Press, Oxford, ISBN 978-0-19-857094-3 (2009)

5. Beall, A.C., Loomis, J.M.: Visual control of steering without course information, Perception, 25, 481-494 (1996)

6. Riemersma,J.B.:Visual control during straight road driving,Acta Psych.,48,215-225 (1981)

7. Macuga,, K.L., Beall, A.C., Kelly, J.W., Smith, R.S., \& Loomis, J.M.: Changing Lanes: inertial information facilitates performance when visual feedback is removed, Experimental Brain Research, 178, 141-150 (2006)

8. Wann, J.P. \& Land, M.F.: Steering with or without the flow: Is the retrieval of heading necessary?, Trends in Cognitive Science, 4, 319-324 (2000)

9. Wann, J.P. \& Wilkie, R.M.: How do we control high-speed steering? In: Vaina, L.M., Beardsley, S.A., \& Rushton, S.K., (eds), Optical Flow and Beyond, pp. 371-389. Kluwer Academic Publishers, Dordrecht (2004) 
Möbus C., Eilers, M. \& Garbe, H., Predicting the Focus of Attention and Deficits in Situation Awareness with a Modular Hierarchical Bayesian Driver Model, in: Vincent G. Duffy (Ed.), Digital Human Modeling, HCI 2011, Orlando, FL, USA

10. Lee,D.N.\&Lishman,R.:Visual control of locomotion,Scand.J.Psych.,18(1),224-230 (1977)

11. Land, M. \& Lee, D.N.: Where we look when we steer, Nature, 369, $742-744$ (1994)

12. Underwood, G., Chapman, P., Crundall, D., Cooper, S., \& Wallen, R.: The visual control of steering and driving: where do we look when negotiating curves? In: Gale, A.G. (ed), Vision in Vehicles VII, pp. 245-252, Elsevier, Amsterdam (1999)

13. Chattington, M., Wilson, M., Ashford, D., \& Maple-Horvat, D.E.: Eye-steering coordination in natural driving, Experimental Brain Research, 180, 1-14 (2007)

14. Mars, F.: Driving around bends with manipulated eye-steering coord.,J.Vision, 8(11) (2008)

15. Kandil, F.I., Rotter, A., \& Lappe, M.: Driving is smoother and more stable when using the tangent point, Journal of Vision, 9(1), 11, 1-11 (2009)

16. Robertshaw, K.D. \& Wilkie, R.M.: Does gaze influence steering around a bend? Journal of Vision, 8(4), 18, 1-13 (2008)

17. Wilkie R. M. \& Wann J. P.: Driving as night falls: The contribution of retinal flow and visual direction to the control of steering. Current Biology, 12, 2014-2017 (2002)

18. Wilkie, R.M. \& Wann, J.P., Eye-Movements Aid the Control of Locomotion, Journal of Vision, 3, 677-684 (2003)

19. Yilmaz, E.R. \& Nakayama, K.: Fluctuation of attention levels during driving, Investigative Ophthalmology and Visual Science, 36, p.940 (1995)

20. Mourant, R.R. \& Rockwell, Th.H.: Mapping Eye-Movement Patterns to the Visual Scene in Driving: An Exploratory Study, Human Factors, Vol. 12(1), 1970 , pp. 81-87

21. Horrey, W.J., Wickens, Ch.D., and Consalus, K.P.: Modeling Driver's Visual Attention Allocation While Interacting With In-Vehicle Technologies, J.Exp.Psych.,12,67-78 (2006)

22. Donges, E.: A 2-level model of driver steering behavior, Hum. Factors, 20,691-707 (1978)

23. Land, M.F.: The Visual Control of Steering, In: L.R. Harris \& M. Jenkin (eds.), Vision and Action, Cambridge University Press, Cambridge, 163-180 (1998)

24. Land, M. et al.: Which Parts of the Road Guide Steering? Nature,377,339-340 (1995)

25. Salvucci, D.D., \& Gray, R.: A Two-Point Visual Control Model of Steering, Perception, $33,1233-1248(2004)$

26. Möbus, C. \& Eilers, M.: Further Steps Towards Driver Modeling according to the Bayesian Programming Approach, In: Duffy, V. (ed), Digital Human Modeling, LNCS (LNAI), Vol. 5620, pp. 413-422, Springer, Heidelberg, ISBN 978-3-642-02808-3 (2009)

27. Möbus, C. \& Eilers, M.: Integrating Anticipatory Competence into a Bayesian Driver Model, In: Cacciabue, C. et al. (ed).: Human Modeling in Assisted Transportation, Springer, HD (in press), ISBN-13: 978-8847018204

28. Möbus, C. \& Eilers, M.: Prototyping Smart Assistance with Bayesian Autonomous Driver Models, In: Chong, N.Y. \& Mastrogiovanni, F. (eds), Handbook of Research on Ambient Intelligence and Smart Environments, IGI Global. (in press), ISBN13: 9781616928575

29. Forbes, T., Kanazawa, H.K., \& Russell, St., The BATmobile: Towards a Bayesian Automated Taxi, IJCAI'95, Vol 2, 1878 -1885 (1995)

30. Eilers, M. \& Möbus, C.: Learning of a Bayesian Autonomous Driver Mixture-ofBehaviors (BAD-MoB) Model, In: Vincent G. Duffy (ed), Advances in Applied Digital Human Modeling, 436-445, CRC Press, Taylor \& Francis Group, Boca Raton, ISBN 9781-4398-3511-1 (2010)

31. Eilers, M. \& Möbus, C.: Learning the Relevant Percepts for Modular Hierarchical Bayesian Driver Models using the Bayesian Information Criterion, HCII (2011, in press)

32. Lee, D.N.: A theory of visual control of braking based on information about time-tocollision. Perception, 5, 437-459 (1976)

33. Lee, D.N.: How Movement is Guided, http://www.perception-in-action.ed.ac.uk/PDF_s/ Howmovementisguided.pdf (2005)

34. Pepping, G.J. \& Grealy, M.A.: Closing the Gap: The Scientific Writings of David N. Lee, Lawrence Erlbaum Associates, Mahwah, N.J., ISBN-13: 978-0805856194 (2007) 